\title{
Impedance Matching Network Based on Metasurfaces (2-D Metamaterials) for Electrically Small Antennas
}

\author{
Mohammad Alibakhshikenari ${ }^{1 *}$, Bal S. Virdee ${ }^{2}$, Chan H. See ${ }^{3,4}$, Raed A. Abd-Alhameed ${ }^{5}$, Francisco \\ Falcone $^{6}$, and Ernesto Limiti ${ }^{1}$

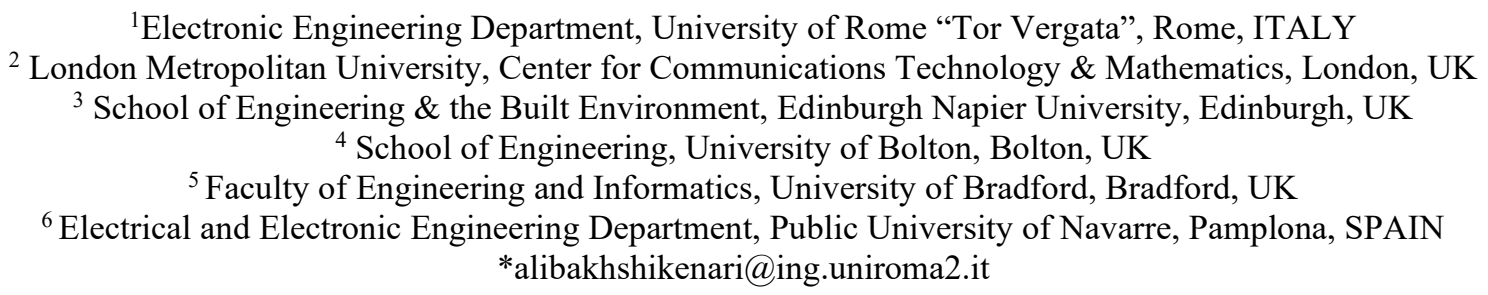

\begin{abstract}
The paper shows the gain-bandwidth limitations of traditional discrete component matching used in electrically small antennas can be overcome by implementing the impedance matching network with metasurface. The proposed metasurface can optimize maximum power transfer to electrically small antennas and achieve superior broadband performance.
\end{abstract}

Keywords- Metasurface impedance matching network, Transmit Antenna, Receive Antenna, signal-to-noise ratio (S/N), RLC components, electrically small antenna, power efficiency.

\section{INTRODUCTION}

With ever shrinking wireless communications systems antennas need to be as small as possible. Electrically small antennas have a reactive impedance, and the magnitude of this reactance is inversely proportional to the antenna's electrical length [1-3]. In addition, it's well known that the radiation resistance is inversely proportional to the square of the antenna's electrical length. It can be deduced that the power factor of the radiation diminishes with the third power of electrical length. Irrespective of geometry and antenna type, electrically small antennas have high-Q [3-9]. The consequence of this is that they are challenging to match, which results in suboptimal power transfer from source to the load, poor radiation efficiency, narrow bandwidth and/or poor gain. At receivers the signal-to-noise $(\mathrm{S} / \mathrm{N})$ ratio is poor compared to using a full-size antenna.

In this paper an impedance matching technique based on metasurface is used to overcome the limitations of gain-bandwidth product. This is achieved by using $L C$ networks of negative inductors and capacitors.

\section{COnVentional Matching Versus Metasurface MATCHING NETWORK}

To match the driving-point impedance of an electrically small antenna to say a $50 \Omega$ system is highly challenging as the antenna will have a high-Q. Even matching with lossless inductors and capacitors is restricted over very narrow bandwidths. However, wideband matching can be achieved by introducing loss in the network, but this will sacrifice efficiency of powertransfer to and from the antenna and will results in low gain performance.

\section{A) Downfall of Low Gain Antenna for a Transmitter \& Receiver}

In transmitter applications, the use of low gain antennas necessitates increase in the transmit power, which is an expensive and undesirable solution. In receivers the low gain antenna results in reduced sensitivity, i.e. poor signal-to-noise ratio.

In [10] the authors have demonstrated impedance matching using metamaterial. The metamaterial was comprised copper spirals deposited on an alumina substrate. The metamaterial structure was designed at 450 MHz. The effectiveness of the metamaterial structure was shown to improve the match of the electrically small loop antenna to the power source impedance which resulted in increase in the overall radiated power.

\section{Proposed Metasurface IMPEdANCE MATCHING NETWORK}

Matching networks implemented with metasurfaces, i.e. a structure that exhibits a negative permittivity and permeability, that are realized with microstrip-lines can achieve a bandwidth of a decade or so centered at the operating frequency. The restrictions imposed by gainbandwidth conditions of conventional passive networks a large antenna prevent them to achieve such a performance.

Maximum power transfer with metasurface matching networks in transmitters will result in greatly enhanced radiation efficiency and gain; and in the case of receivers there should be significant improvement in the signal-tonoise ratio.

Metasurface impedance matching networks can also be applied to phased arrays. This should enable broadband antenna designs and great scan angles.

The proposed metasurface impedance matched network needs to provide a positive phase shift at the antenna and RF front-end circuitry as well as satisfy the characteristic impedance requirements. This has been achieved by means of an artificial-line consisting of a microstrip-line that is loaded with a $2 \mathrm{D}$ metamaterial structure. The metasurface (2D metamaterial) structure is essentially constructed with rectangular spiral resonators, which are connected to the metallic via-holes, as shown in Fig.1. The resonators behave like series left-handed capacitances 
$\left(C_{L}\right)$, and the metallic via-holes play the role of the shunt left-handed inductances $\left(L_{L}\right)$ [11]. The parameters characterizing the geometry of the rectangular spiral resonators and via-holes, i.e. the gap between the lines, the characteristic impedance and length, the relative position of the resonators, and radius of metallic via-holes control its properties.

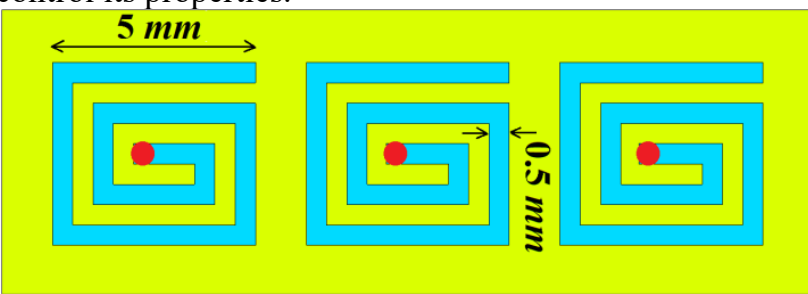

Fig.1. Proposed metasurface constructed with rectangular spiral resonators and via-holes (green area represents the substrate, the blue spirals are metallization etched on the substrate top, and the red circulars are the metallic via-holes).

The analysis on the proposed configuration in Fig.1 has shown it satisfies the required phase and characteristic impedance for necessary to implement the impedancematching network. In the analysis indicates the proposed configuration can be represented by a lumped-element model shown in Fig.2 if the rectangular spiral resonators are electrically small [12]. In this model, for completeness, $C_{L}$ and $L_{L}$ are related to the capacitance and inductance of the rectangular spiral resonators and the metallic via-holes, respectively, according to:

$$
\begin{aligned}
& C_{L}=\frac{L_{L}}{2 \xi^{2} \vartheta^{2}} \\
& L_{L}=2 C_{L} \vartheta^{2} \xi^{2} \\
& \vartheta=\sqrt{ }\left(L_{L} C_{L}\right)
\end{aligned}
$$

where $\xi$ is the coupling between the host line and the rectangular spiral resonators/metallic via-holes, and $\vartheta$ is the resonance frequency of the resonators and via-holes.

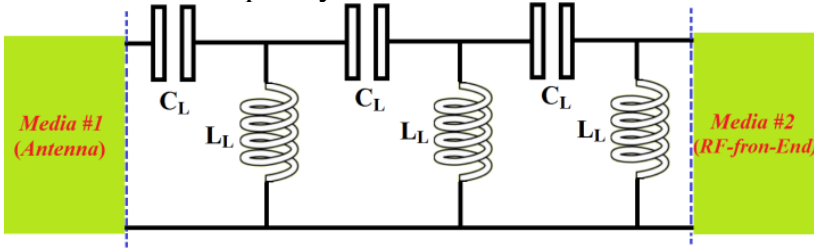

Fig.2. Lumped-element model of the impedance-matching network based on the proposed metasurface (2D metamaterial) shown in Fig.1.

To determine the degree of loss by the structure in Fig.1, a 3D EM simulation was carried out of the propagation through the metasurface impedance matching network. Fig. 3 shows that the loss introduced by the structure is not significant in the frequencies of interest $(0.2 \mathrm{~dB})$. In the response shown in Fig.3(b), the powerloss is an insignificant percentage of the incident power $(5 \%)$ at the frequencies of interest.

\section{CONCLUSIONS}

The proposed metasurface impedance matching structure can be applied to electrically small antennas with significantly less loss resulting from suboptimal matching than would be possible using traditional techniques.

\section{ACKNOWLEDGMENTS}

This work is partially supported by RTI2018-095499B-C31, Funded by Ministerio de Ciencia, Innovación y
Universidades, Gobierno de España (MCIU/AEI/FEDER,UE), and innovation programme under grant agreement H2020-MSCA-ITN-2016 SECRET-722424 and the financial support from the UK Engineering and Physical Sciences Research Council (EPSRC) under grant EP/E022936/1.

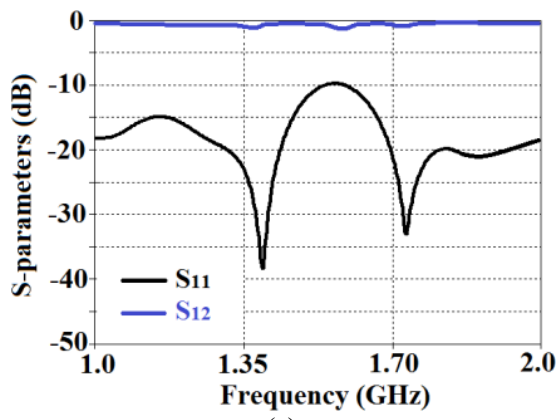

(a)

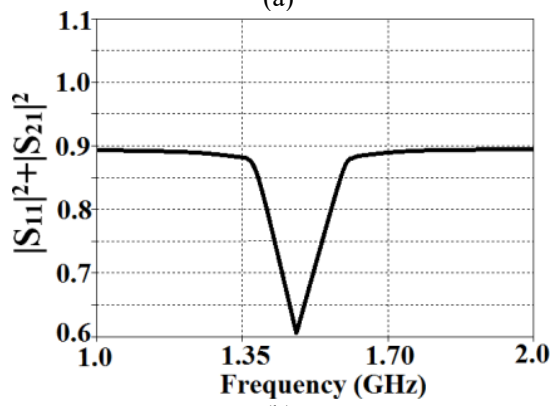

(b)

Fig.3. (a) 3D EM simulation of the S-parameters, and (b) $\left|S_{11}\right|^{2}+\left|S_{21}\right|^{2}$ relation for the structure of Fig. 1.

\section{REFERENCES}

[1] Stephen E. Sussman-Fort, and Ronald M. Rudish, "Non-Foster Impedance Matching of Electrically-Small Antennas", IEEE Transactions on Antennas and Propagation, vol. 57, no. 8, pp. 22302241, August 2009.

[2] Stephen E. Sussman-Fort, "Matching Network Design Using NonFoster Impedances", International Journal of RF and Microwave Computer-Aided Engineering DOI 10.1002/mmce, Volume16, Issue2, March 2006, Pages 135-142.

[3] H. A. Wheeler, "Fundamental limitations of small antennas," Proc. IRE, vol. 35, pp. 1479-1484, Dec. 1947.

[4] L. J. Chu, "Physical limitations of omni-directional antennas," $J$. Appl. Phys., vol. 19, pp. 1163-1175, Dec. 1948.

[5] R. F. Harrington, "Effect of antenna size on gain, bandwidth and efficiency," J. Res. Nat. Bureau Stand., vol. 64D, pp. 1-12, Jan. 1960.

[6] R. C. Hansen, "Fundamental limitations in antennas," Proc. IEEE, vol. 69 , pp. 170-182, Feb. 1981.

[7] J. McLean, "A re-examination of the fundamental limits on the radiation Q of electrically small antennas," IEEE Trans. Antennas Propag., vol. 44, pp. 672-676, May 1996.

[8] W. Geyi and P. Jarmuszewski, "The Foster reactance theorem for antennas and radiation Q," IEEE Trans. Antennas Propag., vol. 48, pp. 401-408, Mar. 2000.

[9] J. C.-E. Sten, A. Hujanen, and P. Koivisto, "Quality factor of an electrically small antenna radiating close to a conducting plane," IEEE Trans. Antennas Propag., vol. 49, pp. 829-837, Mar. 2001.

[10] R. B. Greegor, C. G. Parazzoli, J. A. Nielsen, M. H. Tanielian, D. C. Vier, S. Schultz, Christopher L. Holloway, and Richard W. Ziolkowski, "Demonstration of Impedance Matching Using a muNegative (MNG) Metamaterial," IEEE Antennas and Wireless Propagation Letters, vol. 8, 2009, pp. 92-95.

[11] Christophe Caloz and Tatsuo Itoh, "Electromagnetic Metamaterials: Transmission Line Theory and Microwave Applications", ISBN: 978-0471-66985-2, November 2005, Wiley-IEEE Press, 376 Pages.

[12] Ferran Paredes, Gerard Zamora Gonzàlez, Jordi Bonache, and Ferran Martín,"Dual-Band Impedance-Matching Networks Based on Split-Ring Resonators for Applications in RF Identification (RFID)", IEEE Transactions on Microwave Theory and Techniques, vol. 58, no. 5, pp. 1159-1166, May 2010. 